ARGONNE NATIONAL LABORATORY

9700 South Cass Avenue

Argonne, Illinois 60439

ANL/APS/TB-44

\title{
Guidelines for Beamline Radiation Shielding Design at the Advanced Photon Source
}

\author{
P.K. Job \\ APS Operations Division \\ Advanced Photon Source
}

April 2002

work sponsored by

U.S. DEPARTMENT OF ENERGY

Office of Energy Research 


\title{
Guidelines for Beamline Radiation Shielding Design at the Advanced Photon Source
}

\author{
Compiled by P.K. Job, Radiation Physicist, Advanced Photon Source \\ April 2002
}

\section{Shielding Policy}

Shielding for the APS will be such that the individual worker dose will be ALARA (as low as reasonably achievable) and less than $5 \mathrm{mSv} / \mathrm{yr}(500 \mathrm{mrem} / \mathrm{yr})$. The APS shielding policy requires that the average worker dose be below $2 \mathrm{mSv} / \mathrm{yr}(200 \mathrm{mrem} / \mathrm{yr})$. Worker dose is monitored, and frequent area- surveys are performed by health physics personnel. For cases in which surveys indicate elevated hourly dose rates that may impact worker exposure, additional local shielding is provided to reduce the radiation field to an acceptable level. Passive monitors are used throughout the facility to integrate doses in various areas. The results are analyzed for trends of increased doses, and shielding in these areas is evaluated and improved, as appropriate.

The APS policy for on-site nonradiation workers in the vicinity of the APS facilities requires that the average nonradiation worker dose be below $0.2 \mathrm{mSv} / \mathrm{yr}(20 \mathrm{mrem} / \mathrm{yr})$. In addition, the dose at the site boundary from all pathways is required to be below $0.1 \mathrm{mSv} / \mathrm{yr}(10 \mathrm{mrem} / \mathrm{yr})$. For future modifications of the facility, the doses shall be evaluated, and additional shielding provided to meet the policy requirements.

Shielding guidelines and requirements have been developed and submitted to the collaborative access teams (CATs) to provide the basis for their beamline shielding designs (TB-7, TB-20 and TB-21). These requirements also form the basis for the ionizing radiation hazard analyses described in the Safety Assessment Document of the APS. The Beamline Review Committee will evaluate all shielding designs that do not conform to the criteria presented in the abovereferenced documentation.

The APS policy goal with respect to accidental exposures is to provide enough shielding to mitigate the dose consequence to $<1 \mathrm{mSv}(100 \mathrm{mrem})$ for any one occurrence. Potential accident situations have been analyzed, and the dose consequences evaluated. Additional local shielding has been added at relevant potential loss points to achieve the policy goal. As stated above, when facility modifications are made, the hazard potential will be evaluated and additional shielding supplied, as needed.

\section{Shielding Design Description}

The shielding design for the APS was based on conservative assumptions, including several types of operations that involve normal beam loss mechanisms, as well as certain abnormal beam loss scenarios. These scenarios were drawn from experiences and assumptions used at existing accelerator and synchrotron radiation facilities. The radiation dose limits are less than the DOE guidelines for both on-site and off-site exposures. Design standards for new facilities require that the individual worker dose should be less than $5 \mathrm{mSv} / \mathrm{yr}(500 \mathrm{mrem} / \mathrm{yr})$ and ALARA. This effectively reduces the design dose rate to $2.5 \mu \mathrm{Sv} / \mathrm{h}(0.25 \mathrm{mrem} / \mathrm{h})$ for occupational exposure of 
2000 hours/ year. The shielding is designed to meet or better this criterion to ensure that occupational radiation doses are ALARA.

\section{Shielding Recommendations}

For each shielding situation, the synchrotron and bremsstrahlung shielding have been calculated for a representative geometry. The requirements for both bremsstrahlung and synchrotron radiation are given. The shielding calculations for bremsstrahlung are done by the EGS4 program and for synchrotron radiation by the PHOTON program. All calculations are done at $300 \mathrm{~mA}$ of beam current and $7 \mathrm{GeV}$ electron beam energy. In most cases, one source dominates and the contribution of the other becomes negligible, so the calculated shielding for the dominant source is directly found. The insertion device source spectrum was calculated by the XOP program. For the monochromatic shielding calculations, a conservative bandpass value of $0.1 \%$ was assumed for the monochromator. An appropriate safety factor was added to the final values.

\subsection{Experiment Stations}

Table 1 summarizes the shielding recommendations for the experiment stations at the APS.

\subsubsection{Insertion Device White Beam Stations}

To shield the insertion device (ID) white beam stations and first optics enclosures (FOEs) for synchrotron radiation only requires 19 and $16 \mathrm{~mm}$ of lead for the back wall and for the lateral wall, respectively. The roof will have a very low occupancy rate and will be shielded for 2.5 $\mathrm{mrem} / \mathrm{hr}$, which requires $12 \mathrm{~mm}$ of lead.

The lead thickness for bremsstrahlung only shielding was calculated to be $11.44 \mathrm{~cm}$ at the lowest angle spot on the back wall not shadowed by the tungsten stop. The secondary bremsstrahlung scattering, and hence the required shielding, drops quickly as the angle from the primary beam increases. It is recommended that collimators be placed along the beamline to shadow the back wall from the low-angle scatter of components in the beam. With the use of collimators, the recommended shielding for the entire back wall is $50 \mathrm{~mm}$ of lead. An approximately $1 \mathrm{~m}^{2}$ portion of the back wall, centered on the direct beam, will require an additional $50 \mathrm{~mm}$ of lead. This can also be satisfied with appropriate design of the guillotines.

To summarize, the bremsstrahlung shielding dominates for the back wall, and the synchrotron radiation dominates for the lateral wall and the roof. Lead shielding of $50 \mathrm{~mm}$ is needed for the entire back wall, with an additional $50 \mathrm{~mm}(100 \mathrm{~mm}$ total) for the center portion of the back wall, which can be satisfied with appropriate guillotines. Lead shielding of $19 \mathrm{~mm}$ is needed for the lateral wall, and $12 \mathrm{~mm}$ is needed for the roof. 


\subsubsection{Bending Magnet White Beam Stations}

The bending magnet white beam stations and FOEs require 9, 8, and $6 \mathrm{~mm}$ of lead for the back wall, lateral wall, and roof, respectively, for synchrotron radiation only. The bremsstrahlung for a bending magnet beamline, compared to that for an ID beamline, is reduced by the effective straight section length in the line of sight of the beamline. Dividing the recommended ID shielding thicknesses by an appropriate factor leads to shielding of $9 \mathrm{~mm}$ for the back wall for bremsstrahlung only. An area of approximately $1 \mathrm{~m}^{2}$ around the direct beam at the back wall will require an additional $15 \mathrm{~mm}$ (a total of $24 \mathrm{~mm}$ ) of lead shielding. As with the ID FOE, collimators should be used to reduce the line of sight to the back wall.

In summary, lead thicknesses of $9 \mathrm{~mm}$ for the back wall (24 mm locally), $8 \mathrm{~mm}$ for the lateral wall, and $6 \mathrm{~mm}$ for the roof are recommended for the bending magnet white beam stations.

\subsubsection{Monochromatic Beam Stations}

For the monochromatic station shielding calculations, a conservative bandpass value of $0.1 \%$ was assumed for the monochromator. Most of the radiation that needs to be shielded results from the higher harmonics of the transmitted beam. It is assumed that the bremsstrahlung radiation has been stopped upstream.

The insertion device monochromatic stations require lead shielding of $12.5 \mathrm{~mm}$ for the back (downstream) wall, $10 \mathrm{~mm}$ for the front (upstream) and lateral walls, and $6 \mathrm{~mm}$ for the roof.

The bending magnet monochromatic stations require lead shielding of $7 \mathrm{~mm}$ for the back wall, 6 $\mathrm{mm}$ for the front and lateral walls, and $4 \mathrm{~mm}$ for the roof.

\subsection{Beam Transports}

Table 2 summarizes shielding recommendations for beam transports at the APS.

\subsubsection{White Beam Transport}

It is recommended that, wherever possible, white beam transport be avoided. If deemed necessary, a careful ray trace should be carried out, and collimation should be used to prevent the transport from being hit by the bremsstrahlung or synchrotron radiation beam. Wherever possible, slits, shutters, and any other components that might be struck by the beam should be placed inside an experimental hutch.

To shield the ID beamlines against synchrotron radiation only, $12 \mathrm{~mm}$ of lead transport is needed for air scattering. For a solid scatterer, $21 \mathrm{~mm}$ of lead will be needed for synchrotron radiation only. For the solid scattering of secondary bremsstrahlung, $45 \mathrm{~mm}$ of lead is required in the beam transport outside the beamline enclosures. 
For the bending magnet white beam transport, $6 \mathrm{~mm}$ of lead is needed for air and $11 \mathrm{~mm}$ for solid scatterers for synchrotron radiation only. For the solid scattering of secondary bremsstrahlung, $12 \mathrm{~mm}$ of lead is required in the beam transport outside the beamline enclosures.

\subsubsection{Monochromatic Beam Transport}

For an ID with a $0.1 \%$ bandpass monochromator, the required lead shielding on transport is 8 $\mathrm{mm}$ for vacuum loss scattering. Shielding of $12 \mathrm{~mm}$ lead is recommended for areas in which there are potential solid scatterers.

Likewise, for the bending magnet monobeam transport, the recommended lead shielding for vacuum loss is $5 \mathrm{~mm}$ and for potential solid scatterers is $7 \mathrm{~mm}$.

\subsection{Shutters/Stops}

A shutter is a remotely actuated device, and a stop is a nonmovable, or manually movable, secured device to stop the radiation beam. Table 3 summarizes recommendations for the thickness of shutters/stops at the APS.

\subsubsection{White Beam Shutters/Stops}

All APS shutters (for white and monochromatic beams) are designed to be redundant; two shielding blocks will stop bremsstrahlung and the synchrotron beam when the shutter is closed. Also, the personnel safety interlock system (PSS) will detect any shutter failure through redundant switches and will take appropriate measures to shut off the beam during a fault condition. For stops that are not movable or manually movable and whose mode change is secured with administrative controls, the shielding block need not be redundant.

Synchrotron radiation may place a significant thermal load on beamline components, including shutters and stops. The beamline shall be designed to ensure that thermal protection is provided, if necessary, in order that the shielding cannot be compromised. Such a thermal shutter/stop is located prior to the shielding blocks. The thermal shutter/stop need not be redundant if the water coolant flow/pressure is monitored by the PSS.

The recommended thickness of white beam stops, shutters, and collimators at the APS is $300 \mathrm{~mm}$ of lead or $180 \mathrm{~mm}$ of tungsten. However a 200-mm-thick tungsten block is recommended as an additional safety feature. The transverse dimensions of the shutters/stops are determined from the primary bremsstrahlung ray tracing. The extremal ray in the case of primary bremsstrahlung ray tracing should not be closer than $45 \mathrm{~mm}$ from the lateral edge of the lead or $35 \mathrm{~mm}$ from that of the tungsten shield block. 


\subsubsection{Monochromatic Beam Shutters/Stops}

The thickness of each monochromatic tungsten beam shutter, which is redundant, is specified to be $60 \mathrm{~mm}$ for ID sources and $21 \mathrm{~mm}$ for bending magnet sources. The thickness of monochromatic stops needs to be evaluated based on the monochromatic beam specifications of each beamline. In the monochromatic stations, beam may be stopped on the downstream wall where the thickness of the downstream wall is greater than or equal to the sum of the combined thickness required of the stop and the downstream wall of the station.

The pink beam components in the beamlines (stations, shutters, and stops) have the same shielding requirements as do monochromatic beam components. However for thermal load considerations, the white beam case applies.

In the case of a white beam stop, monobeam pass through, tungsten integral shutters for the monochromatic beam aperture, an offset of $8 \mathrm{~mm}$ or more is safe for the extremal bremsstrahlung ray from the edge of the monoaperture to minimize the cross talk. For the lead apertures, this offset is $15 \mathrm{~mm}$. 
Table 1. Shielding recommendations for experiment stations (Pb thickness)

\begin{tabular}{|l|c|c|c|c|}
\hline \multicolumn{1}{|c|}{ Station Type } & Upstream Panel & Lateral Panel & Roof & $\begin{array}{c}\text { Downstream } \\
\text { Panel }\end{array}$ \\
\hline ID White Beam & $19 \mathrm{~mm}$ & $19 \mathrm{~mm}$ & $12 \mathrm{~mm}$ & $\begin{array}{c}50 \mathrm{~mm} \\
(100 \text { locally })\end{array}$ \\
\hline BM White Beam & $8 \mathrm{~mm}$ & $8 \mathrm{~mm}$ & $6 \mathrm{~mm}$ & $\begin{array}{c}9 \mathrm{~mm} \\
(24 \text { locally })\end{array}$ \\
\hline ID Mono/Pink Beam & $10 \mathrm{~mm}$ & $10 \mathrm{~mm}$ & $6 \mathrm{~mm}$ & $12 \mathrm{~mm}$ \\
\hline BM Mono/Pink Beam & $6 \mathrm{~mm}$ & $6 \mathrm{~mm}$ & $4 \mathrm{~mm}$ & $7 \mathrm{~mm}$ \\
\hline
\end{tabular}

Table 2. Shielding recommendations for beam transport ( $\mathrm{Pb}$ thickness)

\begin{tabular}{|l|c|c|c|}
\hline \multicolumn{1}{|c|}{ Beam Type } & Air as Scatterer & $\begin{array}{c}\text { Solid Scatterer } \\
\text { (Synch. Radiation) }\end{array}$ & $\begin{array}{c}\text { Solid Scatterer } \\
\text { (Bremsstrahlung) }\end{array}$ \\
\hline ID White Beam & $12 \mathrm{~mm}$ & $21 \mathrm{~mm}$ & $45 \mathrm{~mm}$ \\
\hline BM White Beam & $6 \mathrm{~mm}$ & $11 \mathrm{~mm}$ & $12 \mathrm{~mm}$ \\
\hline ID Mono/Pink Beam & $8 \mathrm{~mm}$ & $12 \mathrm{~mm}$ & \\
\hline BM Mono/Pink Beam & $5 \mathrm{~mm}$ & $7 \mathrm{~mm}$ & \\
\hline
\end{tabular}

Table 3. Recommendations for shutter/stop thickness

\begin{tabular}{|l|c|c|}
\hline \multicolumn{1}{|c|}{ Beam Type } & Tungsten Thickness & Lead Thickness \\
\hline ID White Beam & $180 \mathrm{~mm}^{(\mathrm{a})}$ & $300 \mathrm{~mm}$ \\
\hline BM White Beam & $180 \mathrm{~mm}^{(\mathrm{a})}$ & $300 \mathrm{~mm}$ \\
\hline ID Mono/Pink Beam & $60 \mathrm{~mm}$ & \\
\hline BM Mono/Pink Beam & $21 \mathrm{~mm}$ & \\
\hline
\end{tabular}

(a) However $200 \mathrm{~mm}$ is recommended as an additional safety feature 